\title{
BOUNDARY VALUE PROBLEMS FOR SECOND AND HIGHER ORDER DIFFERENTIAL EQUATIONS
}

\author{
DÓNAL O'REGAN \\ (Communicated by Kenneth R. Meyer)
}

\begin{abstract}
In this paper the nonlinear alternative of Leray-Schauder is used to obtain existence results for second and higher order boundary value problems.
\end{abstract}

\section{INTRODUCTION}

The purpose of this paper is to provide existence results for $k$ th order boundary value problems. We begin in $\S 2$ by examining the two-point boundary value problem

$$
y^{\prime \prime}(t)=f\left(t, y(t), y^{\prime}(t)\right), \quad 0<t<1,
$$

where $f:[0,1] \times \mathbb{R}^{2} \rightarrow \mathbb{R}$ is continuous and with $y$ satisfying either

(i) $y(0)=A, y(1)=B$

(ii) $y(0)=A, p y(1)+q y^{\prime}(1)=B ; p, q>0$;

(iii) $y(1)=B, r y(0)-s y^{\prime}(0)=A ; r, s,>0$;

(iv) $y(0)=A, y^{\prime}(1)=B$

(v) $y^{\prime}(0)=A, y(1)=B$; or

(vi) $r y(0)-s y^{\prime}(0)=A, p y(1)+q y^{\prime}(1)=B ; r, s, p, q>0$.

The above problem has been studied by a variety of authors with the most advanced results to date being $[2,3,5-7,11-13]$; however, the results of this paper are not only new, they improve, extend, and complement the existing theory. Moreover, the results trivially carry over to systems of second order boundary value problems. Boundary value problems of the above type occur frequently in mathematical physics. For example, a steady state temperature distribution $y$ in a rod (identified with the closed unit interval) is governed by the differential equation $\left(k y^{\prime}\right)^{\prime}+q(t, y)=0$ where $k=k(t, y)>0$ is the thermal conductivity at position $t$ and temperature $y$ and $q(t, y)$ describes interval heat sources. The differential equation may be expressed as $y^{\prime \prime}=$ $f\left(t, y, y^{\prime}\right)$ where $f(t, y, p)=-\frac{1}{k}\left[k_{t} p+k_{y} p^{2}+q(t, y)\right]$.

In addition to techniques and ideas $\S 2$ also provides a method for the study of higher order $k$-point boundary value problems. To indicate the strategy we

Received by the editors April 28, 1990.

1980 Mathematics Subject Classification (1985 Revision). Primary 34B15. 
consider in $\S 3$ a nonlinear fourth order two-point boundary value problem:

$$
\left\{\begin{array}{l}
y^{\text {iv }}(t)=f\left(t, y(t), y^{\prime}(t), y^{\prime \prime}(t), y^{\prime \prime \prime}(t)\right), \quad 0<t<1 \\
y(0)=A, y(1)=B, y^{\prime \prime}(0)=C, y^{\prime \prime}(1)=D
\end{array}\right.
$$

which arises in the theory of elastic beams. For example, the equation describing the deformation of an elastic beam is given by $y^{i v}(t)+f(t) y(t)=g(t), 0<t<1$ and if both ends are simply supported we have $y(0)=y(1)=y^{\prime \prime}(0)=y^{\prime \prime}(1)=$ 0 .

The existence discussion is based on a Nonlinear Alternative of LeraySchauder $[4,7,8]$.

Theorem (Nonlinear Alternative). Assume that $U$ is a relatively open subset of a convex set $K$ in a Banach space $E$. Let $N: \bar{U} \rightarrow K$ be a compact map and assume $p \in U$. Then either

(i) $N$ has a fixed point in $\bar{U}$; or

(ii) there is a $u \in U$ and $\lambda \in(0,1)$ such that $u=\lambda N u+(1-\lambda) p$.

Consider the problem

$$
y^{(k)}=f\left(t, y, y^{\prime}, \ldots, y^{(k-1)}\right), \quad 0<t<1 ; y \in \mathscr{B},
$$

where $\mathscr{B}$ denotes a set of linear homogeneous or affine boundary conditions. Also let $C_{\mathscr{B}}^{k}[0,1]$ be those functions in $C^{k}[0,1]$ that satisfy the boundary condition $\mathscr{B}$. Associated with (1.1) introduce a family of problems

$$
y^{(k)}=\lambda f\left(t, y, y^{\prime}, \ldots, y^{(k-1)}\right), \quad y \in \mathscr{B},
$$

where $0<\lambda<1$. Replacing the differential equation (1.2) with an equivalent Volterra integral equation and applying the Nonlinear Alternative we immediately obtain (this is a restatement of [7, Corollary 2.3; 8, Theorem 3.1]).

Theorem 1.1. Let $f:[0,1] \times \mathbb{R}^{k} \rightarrow \mathbb{R}$ be continuous and $L: C_{\mathscr{B}}^{k}[0,1] \rightarrow C[0,1]$ is one-to-one; here $L$ is the differential operator $L y=y^{(k)}$. Suppose there exists a constant $M$ such that $|y|_{k-1}<M$ for each solution $y$ to (1.2) for each $\lambda \in(0,1)$; here $|y|_{k-1}=\max \left\{|y|_{0}, \ldots,\left|y^{(k-1)}\right|_{0}\right\}$ where

$$
|y|_{0}=\max \{|y(t)|: 0 \leq t \leq 1\} \text {. }
$$

Then (1.1) has at least one solution in $C^{k}[0,1]$.

Our analysis therefore reduces to obtaining a priori bounds independent of $\lambda$ for solutions $y$ (and their first $k-1$ derivatives) to (1.2). For convenience we gather together some of the inequalities we will use in the forthcoming sections.

Theorem 1.2.

(a) Suppose $u \in C^{1}[a, b]$ with $u(a)=0$. Then

$$
\|u\|^{2} \leq \frac{4(b-a)^{2}}{\pi^{2}}\left\|u^{\prime}\right\|^{2} \text {. }
$$

Here $\|u\|^{2}=\int_{a}^{b}|u(t)|^{2} d t$. 
(b) (Wirtinger's Inequality) Suppose $u \in C^{1}[a, b]$ with $u(a)=u(b)=0$. Then

$$
\|u\|^{2} \leq \frac{(b-a)^{2}}{\pi^{2}}\left\|u^{\prime}\right\|^{2} .
$$

(c) Suppose $u \in C^{3}[0,1]$ with $u(0)=u(1)=u^{\prime \prime}(0)=u^{\prime \prime}(1)=0$. Then

$$
\left\|u^{\prime}\right\| \leq \frac{1}{\pi^{2}}\left\|u^{\prime \prime \prime}\right\| \text {. }
$$

Proof. For a proof of (a) and (b) see [10]. To prove (c) recall that Wirtinger's inequality implies $\|u\| \leq \frac{1}{\pi}\left\|u^{\prime}\right\|$ and $\left\|u^{\prime \prime}\right\| \leq \frac{1}{\pi}\left\|u^{\prime \prime \prime}\right\|$. Then integration by parts and Hölder's integral inequality implies

$$
\left\|u^{\prime}\right\|^{2}=-\int_{0}^{1} u(t) u^{\prime \prime}(t) d t \leq\|u\|\left\|u^{\prime \prime}\right\| \leq \frac{1}{\pi}\left\|u^{\prime}\right\| \frac{1}{\pi}\left\|u^{\prime \prime \prime}\right\|
$$

and the result follows.

\section{SECOND ORDER EQUATIONS}

In this section we establish general existence results for problems of the form $y^{\prime \prime}=f\left(t, y, y^{\prime}\right), 0<t<1$, where $y$ satisfies a variety of boundary conditions. First consider the two-point "Dirichlet" boundary value problem:

$$
\left\{\begin{array}{l}
y^{\prime \prime}(t)=f\left(t, y(t), y^{\prime}(t)\right), \quad 0<t<1, \\
y(0)=A, y(1)=B,
\end{array}\right.
$$

where $f:[0,1] \times \mathbb{R}^{2} \rightarrow \mathbb{R}$ is continuous. Making the change of variables $w(t)=$ $y(t)-\mu(t)$, where $\mu(t)=(B-A) t+A$, we see that if $y(t)$ is a solution to (2.1) then $w(t)$ satisfies

$$
\left\{\begin{array}{l}
w^{\prime \prime}(t)=f\left(t, w(t)+\mu(t), w^{\prime}(t)+\mu^{\prime}(t)\right), \quad 0<t<1, \\
w(0)=0, w(1)=0 .
\end{array}\right.
$$

The idea is to show that (2.2) has a solution. Once this is achieved then automatically (2.1) has a solution.

Theorem 2.1. Assume that $f$ has the decomposition $f(t, u, v)=g(t, u, v)+$ $h(t, u, v)$ such that

(i) $u g(t, u, v) \geq a u^{2}+b u v+c|u v|$ for $(t, u, v) \in[0,1] \times \mathbb{R}^{2}$ and

$$
|g(t, u, v)| \leq C(t, u)|v|^{2}+D(t, u)
$$

where $C$ and $D$ are bounded on bounded sets.

(ii) There exists constants $K \geq 0,0 \leq m<2,0 \leq n<2$ such that $\mu(t) g(t, u, v) \leq K\left\{|u|^{m}+|v|^{n}+1\right\}$ for $(t, u, v) \in[0,1] \times \mathbb{R}^{2}$.

(iii) $|h(t, u, v)| \leq M\left\{|u|^{\alpha}+|v|^{\beta}+1\right\}$ for constants $M, \alpha$, and $\beta$ with $0 \leq \alpha, \beta<1$. 
Then (2.2) (and consequently (2.1)) has a $C^{2}[0,1]$ solution in each of the following cases:

(*) $a \geq 0,|c|<\pi$

(**) $a<0,|c| \pi<\pi^{2}+a$.

Proof. By Theorem 1.1 existence of a solution to (2.2) will follow once a priori bounds in $C^{1}[0,1]$ are established for solutions $w(t)$ to

$$
\left\{\begin{array}{l}
w^{\prime \prime}=\lambda f\left(t, w+\mu, w^{\prime}+\mu^{\prime}\right), \quad 0<t<1, \\
w(0)=0, w(1)=0,
\end{array}\right.
$$

where $0<\lambda<1$. Firstly, integration by parts yields $\int_{0}^{1} w(t) w^{\prime \prime}(t) d t=-\left\|w^{\prime}\right\|^{2}$ and this together with the differential equation implies

$$
\begin{aligned}
\left|w^{\prime}\right|^{2} \leq & -\lambda \int_{0}^{1}(w+\mu) g\left(t, w+\mu, w^{\prime}+\mu^{\prime}\right) d t \\
& +\lambda \int_{0}^{1} \mu g\left(t, w+\mu, w^{\prime}+\mu^{\prime}\right) d t+\int_{0}^{1}|w|\left|h\left(t, w+\mu, w^{\prime}+\mu^{\prime}\right)\right| d t .
\end{aligned}
$$

Cauchy's inequality $a b \leq \varepsilon a^{2} / 2+b^{2} /(2 \varepsilon)$ yields

$$
\int_{0}^{1}|w|\left|h\left(t, w+\mu, w^{\prime}+\mu^{\prime}\right)\right| d t \leq \frac{\varepsilon}{2}\|w\|^{2}+\frac{1}{2 \varepsilon} \int_{0}^{1}\left|h\left(t, w+\mu, w^{\prime}+\mu^{\prime}\right)\right|^{2} d t .
$$

Condition (iii) also implies

$$
\begin{aligned}
\left|h\left(t, w+\mu, w^{\prime}+\mu^{\prime}\right)\right|^{2} & \leq 4 M^{2}\left\{|w(t)+\mu(t)|^{2 \alpha}+\left|w^{\prime}(t)+\mu^{\prime}(t)\right|^{2 \beta}+1\right\} \\
& \leq 4 M^{2}\left\{2^{2 \alpha}|w(t)|^{2 \alpha}+2^{2 \beta}\left|w^{\prime}(t)\right|^{2 \beta}+C_{1}\right\}
\end{aligned}
$$

for some constant $C_{1}$ (independent of $\lambda$ ). Now Hölder's integral inequality gives for any $\delta \in[0,1]$

$$
\int_{0}^{1}|w(t)|^{2 \delta} d t \leq\left\{\int_{0}^{1}|w(t)|^{2} d t\right\}^{\delta}=\|w\|^{2 \delta}
$$

and consequently,

$$
\begin{aligned}
& \int_{0}^{1}|w|\left|h\left(t, w+\mu, w^{\prime}+\mu^{\prime}\right)\right| d t \\
& \quad \leq \frac{\varepsilon}{2}\|w\|^{2}+\frac{2 M^{2}}{\varepsilon}\left\{2^{2 \alpha}\|w\|^{2 \alpha}+2^{2 \beta}\left\|w^{\prime}\right\|^{2 \beta}+C_{1}\right\} .
\end{aligned}
$$

On the other hand, Hölder's inequality also gives

$$
\int_{0}^{1}|w(t)|\left|w^{\prime}(t)\right| d t \leq\|w\|\left\|w^{\prime}\right\| ; \quad \int_{0}^{1}|w(t)| d t \leq\|w\|
$$


and so condition (i) and (ii) imply

$$
\begin{aligned}
G(\lambda) \equiv & -\lambda \int_{0}^{1}(w+\mu) g\left(t, w+\mu, w^{\prime}+\mu^{\prime}\right) d t+\lambda \int_{0}^{1} \mu g\left(t, w+\mu, w^{\prime}+\mu^{\prime}\right) d t \\
\leq & -a \lambda \int_{0}^{1}(w+\mu)^{2} d t-b \lambda \int_{0}^{1}(w+\mu)\left(w^{\prime}+\mu^{\prime}\right) d t \\
& +|c| \int_{0}^{1}|w+\mu|\left|w^{\prime}+\mu^{\prime}\right| d t \\
& +K\left\{\int_{0}^{1}|w+\mu|^{m} d t+\int_{0}^{1}\left|w^{\prime}+\mu^{\prime}\right|^{n} d t+1\right\} \\
\leq & -a \lambda\|w\|^{2}+2|a| K_{0}\|w\|+|a| K_{0}^{2}+|b|\left\{K_{1}\|w\|+K_{0}\left\|w^{\prime}\right\|+K_{0} K_{1}\right\} \\
& +|c|\left\{\|w\|\left\|w^{\prime}\right\|+K_{0}\left\|w^{\prime}\right\|+K_{1}\|w\|+K_{0} K_{1}\right\} \\
& +K\left\{2^{m}\|w\|^{m}+2^{n}\left\|w^{\prime}\right\|^{n}+C_{2}\right\}
\end{aligned}
$$

for some constant $C_{2}$; here $K_{j}=\max _{[0,1]}\left|\mu^{(j)}(t)\right| ; j=0,1$. Let $E=$ $2|a| K_{0}+|b| K_{1}+|c| K_{1}, \quad F=|b| K_{0}+|c| K_{0}, \quad H=a K_{0}^{2}+|b| K_{0} K_{1}+|c| K_{0} K_{1}$ and consequently,

$$
\begin{aligned}
G(\lambda) \leq & -a \lambda\|w\|^{2}+E\|w\|+F\left\|w^{\prime}\right\|+H+|c|\|w\|\left\|w^{\prime}\right\| \\
& +K\left\{2^{m}\|w\|^{m}+2^{n}\left\|w^{\prime}\right\|^{n}+C_{2}\right\}
\end{aligned}
$$

Substitute (2.4) and (2.5) into (2.3) to obtain

$$
\begin{aligned}
\left\|w^{\prime}\right\|^{2} \leq & a \lambda\|w\|^{2}+E\|w\|+F\left\|w^{\prime}\right\|+H+|c|\|w\|\left\|w^{\prime}\right\|+\frac{\varepsilon}{2}\|w\|^{2} \\
& +K\left\{2^{m}\|w\|^{m}+2^{n}\left\|w^{\prime}\right\|^{n}+C_{2}\right\} \\
& +\frac{2 M^{2}}{\varepsilon}\left\{2^{2 \alpha}\|w\|^{2 \alpha}+2^{2 \beta}\left\|w^{\prime}\right\|^{2 \beta}+C_{2}\right\} .
\end{aligned}
$$

Wirtinger's inequality yields

$$
\begin{aligned}
\left(1-\frac{|c|}{\pi}-\frac{\varepsilon}{2 \pi^{2}}\right)\left\|w^{\prime}\right\|^{2} \leq & -a \lambda\|w\|^{2}+\left(\frac{E}{\pi}+F\right)\left\|w^{\prime}\right\|+H \\
& +K\left\{\left(\frac{2}{\pi}\right)^{m}\left\|w^{\prime}\right\|^{m}+2^{n}\left\|w^{\prime}\right\|^{n}+C_{2}\right\} \\
& +\frac{2 M^{2}}{\varepsilon}\left\{\left(\frac{2}{\pi}\right)^{2 \alpha}\left\|w^{\prime}\right\|^{2 \alpha}+2^{2 \beta}\left\|w^{\prime}\right\|^{2 \beta}+C_{2}\right\} .
\end{aligned}
$$

Consider case $(*)$ with $a \geq 0$ and $|c|<\pi$. With these assumptions we can delete the $a \lambda$ term from $(2.6)$ and fix $\varepsilon>0$ suitably small so that the term in brackets on the left-hand side of (2.6) is positive. This done, (2.6) and $\alpha, \beta<1, m, n<2$ implies that there exists a constant $M_{1}$ independent of $\lambda$ such that

$$
\left\|w^{\prime}\right\| \leq M_{1}
$$

for any solution $w(t)$ to $\left(2.2_{\lambda}\right)$. 
Now consider the case $(* *)$ with $a<0$ and $|c| \pi<\pi^{2}+a$ i.e. $1-|c| / \pi+$ $a / \pi^{2}>0$. Since $a<0$ we have $-a \lambda \leq-a$ and so $-a \lambda\|w\|^{2} \leq-a\|w\|^{2} \leq$ $-\left(a / \pi^{2}\right)\left\|w^{\prime}\right\|^{2}$. Substitute this into (2.6) to obtain on the left-hand side

$$
\left(1-\frac{|c|}{\pi}+\frac{a}{\pi^{2}}-\frac{\varepsilon}{2 \pi^{2}}\right)\left\|w^{\prime}\right\|^{2} \text {. }
$$

Now fix $\varepsilon>0$ suitably small so that the term in brackets is positive. As above this leads to (2.7) for a certain constant $M_{1}$ independent of $\lambda$ in $(0,1)$.

From (2.7) with $w(0)=0$ we find that $|w(t)| \leq \int_{0}^{1}\left|w^{\prime}(s)\right| d s \leq\left\|w^{\prime}\right\| \leq M_{1}$ for all $t \in[0,1]$, which is the required a priori bound on $w(t)$. Given this bound with (i), (ii), and (iii), we can determine constants $I$ and $J$ (independent of $\lambda$ ) such that

$$
\left|f\left(t, w+\mu, w^{\prime}+\mu^{\prime}\right)\right| \leq I\left|w^{\prime}\right|^{2}+J .
$$

Consequently

$$
\int_{0}^{1}\left|w^{\prime \prime}(t)\right| d t \leq I \int_{0}^{1}\left|w^{\prime}(t)\right|^{2} d t+J \leq I\left\|w^{\prime}\right\|^{2}+J \leq I M_{1}^{2}+J \equiv M_{2} .
$$

Also since $w(0)=w(1)=0$ we have $w^{\prime}(\xi)=0$ for some $\xi \in(0,1)$. Thus

$$
\left|w^{\prime}(t)\right| \leq \int_{0}^{1}\left|w^{\prime \prime}(t)\right| d t \leq M_{2} \text { for } t \in[0,1] .
$$

All the required a priori bounds are obtained and a solution to (2.2) exists.

We next consider the one-sided Sturm-Liouville problem:

$$
\left\{\begin{array}{l}
y^{\prime \prime}(t)=f\left(t, y(t), y^{\prime}(t)\right), \quad 0<t<1, \\
y(0)=A, p y(1)+q y^{\prime}(1)=B ; \quad p, q>0,
\end{array}\right.
$$

where $f:[0,1] \times \mathbb{R}^{2} \rightarrow \mathbb{R}$ is continuous. Making the change of variables $w(t)=$ $y(t)-A$ we see that if $y(t)$ is a solution to $(2.8)$, then $w(t)$ satisfies

$$
\left\{\begin{array}{l}
w^{\prime \prime}(t)=f\left(t, w(t)+A, w^{\prime}(t)\right), \quad 0<t<1, \\
w(0)=0, p w(1)+q w^{\prime}(1)=B-p A
\end{array}\right.
$$

Theorem 2.2. Suppose conditions (i), with $b=0$, and (iii) of Theorem 2.1 are satisfied. In addition suppose

(ii)* there exists constants $K \geq 0,0 \leq m<2,0 \leq n<2$ such that $A g(t, u, v) \leq K\left\{|u|^{m}+|v|^{n}+1\right\}$ for $(t, u, v) \in[0,1] \times \mathbb{R}^{2}$.

Then (2.9) (and consequently (2.8)) has a $C^{2}[0,1]$ solution in each of the following cases:

(*) $a \geq 0,|c|<\pi / 2$;

(**) $a<0,|c| \pi<\pi^{2} / 2+2 a$. 
Proof. Let $w(t)$ be a solution to

$$
\left\{\begin{array}{l}
w^{\prime \prime}(t)=\lambda f\left(t, w(t)+A, w^{\prime}(t)\right), \quad 0<t<1, \\
w(0)=0, p w(1)+q w^{\prime}(1)=B-p A
\end{array}\right.
$$

where $0<\lambda<1$. Integration by parts yields

$$
\begin{aligned}
\int_{0}^{1} w(t) w^{\prime \prime}(t) d t & =\frac{-p}{q} w^{2}(1)+\left(\frac{B-p A}{q}\right) w(1)-\left\|w^{\prime}\right\|^{2} \\
& \leq\left(\frac{B-p A}{q}\right) w(1)-\left\|w^{\prime}\right\|^{2}
\end{aligned}
$$

and this together with the differential equation implies

$$
\begin{aligned}
\left\|w^{\prime}\right\|^{2} \leq & \left(\frac{B-A p}{q}\right) w(1)-\lambda \int_{0}^{1}(w+A) g\left(t, w+A, w^{\prime}\right) d t \\
& +\lambda A \int_{0}^{1} g\left(t, w+A, w^{\prime}\right) d t+\int_{0}^{1}|w|\left|h\left(t, w+A, w^{\prime}\right)\right| d t \\
\leq & \left(\frac{|B|+|A| p}{q}\right)\left\|w^{\prime}\right\|-\lambda \int_{0}^{1}(w+A) g\left(t, w+A, w^{\prime}\right) d t \\
& +\lambda A \int_{0}^{1} g\left(t, w+A, w^{\prime}\right) d t+\int_{0}^{1}|w|\left|h\left(t, w+A, w^{\prime}\right)\right| d t .
\end{aligned}
$$

The result follows by essentially the same reasoning as in Theorem 2.1.

Remark. Similar existence results may be obtained for $y^{\prime \prime}(t)=f\left(t, y(t), y^{\prime}(t)\right)$, $0<t<1$ with the boundary conditions being either

(a) $y(1)=B, r y(0)-s y^{\prime}(0)=A ; r, s>0$;

(b) $y(0)=A, y^{\prime}(1)=B$; or

(c) $y^{\prime}(0)=A, y(1)=B$.

Finally, in this section we examine the relatively unexplored pure SturmLiouville problem:

$$
\left\{\begin{array}{l}
y^{\prime \prime}(t)=f\left(t, y(t), y^{\prime}(t)\right), \quad 0<t<1, \\
r y(0)-s y^{\prime}(0)=A ; \quad r, s>0, \\
p y(1)+q y^{\prime}(1)=B ; \quad p, q>0
\end{array}\right.
$$

with its related family of problems

$$
\left\{\begin{array}{l}
y^{\prime \prime}(t)=\lambda f\left(t, y(t), y^{\prime}(t)\right), \quad 0<t<1, \\
r y(0)-s y^{\prime}(0)=A \\
p y(1)+q y^{\prime}(1)=B
\end{array}\right.
$$

In the process of proving the existence of a solution to $(2.10)$ we will need the following lemmas.

Lemma 2.3. For any function $y \in C^{1}[0,1]$

(i) $\|y\| \leq \frac{2}{\pi}\left\|y^{\prime}\right\|+|y(0)|$;

(ii) $\|y\| \leq \frac{2}{\pi}\left\|y^{\prime}\right\|+|y(1)|$; and

(iii) $\|y\| \leq \frac{2}{\pi}\left\|y^{\prime}\right\|+\frac{1}{2}|y(0)|+\frac{1}{2}|y(1)|$. 
Proof. (i) Let $u(t)=y(t)-y(0)$. Then $u(0)=0$ so Theorem 1.2(a) implies $\int_{0}^{1}[y(t)-y(0)]^{2} d t \leq\left(4 / \pi^{2}\right)\left\|y^{\prime}\right\|^{2}$, i.e.

$$
\|y\|^{2}-2 y(0) \int_{0}^{1} y(t) d t+y^{2}(0) \leq \frac{4}{\pi^{2}}\left\|y^{\prime}\right\|^{2} .
$$

Now Hölder's inequality implies

$$
\|y\|^{2}-2|y(0)|\|y\|+y^{2}(0) \leq \frac{4}{\pi^{2}}\left\|y^{\prime}\right\|^{2} .
$$

That is $\{\|y\|-|y(0)|\}^{2} \leq\left(4 / \pi^{2}\right)\left\|y^{\prime}\right\|^{2}$ and the result follows.

(ii) Follows the ideas of (i) with $v(t)=y(t)-y(1)$.

(iii) Combining (i) and (ii) gives the result.

Lemma 2.4. For any function $y \in C^{1}[0,1]$

$$
\|y\|^{2} \leq\left(\frac{\sqrt{2}}{\sqrt{2}-1}\right) \frac{1}{\pi^{2}}\left\|y^{\prime}\right\|^{2}+\left(\frac{2-\sqrt{2}}{2(\sqrt{2}-1)}\right)\left[y^{2}(0)+y^{2}(1)\right] .
$$

Proof. Theorem 1.2(a) implies

$$
\begin{aligned}
& \int_{0}^{\frac{1}{2}}(y(t)-y(0))^{2} d t \leq \frac{1}{\pi^{2}} \int_{0}^{\frac{1}{2}}\left(y^{\prime}(t)\right)^{2} d t ; \\
& \int_{\frac{1}{2}}^{1}(y(t)-y(1))^{2} d t \leq \frac{1}{\pi^{2}} \int_{\frac{1}{2}}^{1}\left(y^{\prime}(t)\right)^{2} d t .
\end{aligned}
$$

This together with Hölder's inequality implies

$$
\begin{aligned}
\|y\|^{2} \leq & \frac{1}{\pi^{2}}\left\|y^{\prime}\right\|^{2}+2 y(0) \int_{0}^{\frac{1}{2}} y(t) d t+2 y(1) \int_{\frac{1}{2}}^{1} y(t) d t-\frac{1}{2}\left[y^{2}(0)+y^{2}(1)\right] \\
\leq & \frac{1}{\pi^{2}}\left\|y^{\prime}\right\|^{2}+\frac{2}{\sqrt{2}}|y(0)|\left\{\int_{0}^{\frac{1}{2}} y^{2}(t) d t\right\}^{\frac{1}{2}}+\frac{2}{\sqrt{2}}|y(1)|\left\{\int_{\frac{1}{2}}^{1} y^{2}(t) d t\right\}^{\frac{1}{2}} \\
& -\frac{1}{2}\left[y^{2}(0)+y^{2}(1)\right] \\
\leq & \frac{1}{\pi^{2}}\left\|y^{\prime}\right\|^{2}+\frac{1}{\sqrt{2}}\left[y^{2}(0)+\int_{0}^{\frac{1}{2}} y^{2}(t) d t\right]+\frac{1}{\sqrt{2}}\left[y^{2}(1)+\int_{\frac{1}{2}}^{1} y^{2}(t) d t\right] \\
& -\frac{1}{2}\left[y^{2}(0)+y^{2}(1)\right] \\
= & \frac{1}{\pi^{2}}\left\|y^{\prime}\right\|^{2}+\frac{1}{\sqrt{2}}\|y\|^{2}+\frac{2-\sqrt{2}}{2 \sqrt{2}}\left[y^{2}(0)+y^{2}(1)\right] .
\end{aligned}
$$

The result now follows.

Theorem 2.5. Assume $f$ has the decomposition $f(t, u, v)=g(t, u, v)+$ $h(t, u, v)$ with

(i) $u g(t, u, v) \geq a u^{2}+b|u v|$ for $(t, u, v) \in[0,1] \times \mathbb{R}^{2}$ and $|g(t, u, v)| \leq$ $C(t, u)|v|^{2}+D(t, u)$ where $C$ and $D$ are bounded on bounded sets; 
(ii) $|h(t, u, v)| \leq M\left\{|u|^{\alpha}+|v|^{\beta}+1\right\}$ for constants $M, \alpha$ and $\beta$ with $0 \leq$ $\alpha, \beta<1$.

Then (2.10) has a $C^{2}[0,1]$ solution in each of the following cases:

(*) $a \geq 0,|b|<\min \left\{\frac{2 \pi}{4+\pi}, \frac{4 p}{q}, \frac{4 r}{s}\right\}$,

$(* *) \quad a<0,|b|<\min \left\{\frac{2 \pi}{4+\pi}+\frac{2 \sqrt{2} a}{\pi(4+\pi)(\sqrt{2}-1)}, \frac{4 p}{q}+\frac{2 a(2-\sqrt{2})}{\sqrt{2}-1}, \frac{4 r}{s}+\frac{2 a(2-\sqrt{2})}{\sqrt{2}-1}\right\}$.

Proof. Let $y(t)$ be a solution to $\left(2.10_{\lambda}\right)$ so integration by parts together with the boundary conditions yield

$$
\begin{aligned}
\int_{0}^{1} y(t) y^{\prime \prime}(t) d t & =y(1) y^{\prime}(1)-y(0) y^{\prime}(0)-\left\|y^{\prime}\right\|^{2} \\
& =-\left\|y^{\prime}\right\|^{2}-\frac{p}{q} y^{2}(1)-\frac{r}{s} y^{2}(0)+\frac{B}{q} y(1)+\frac{A}{s} y(0) .
\end{aligned}
$$

Consequently

$$
\begin{aligned}
\left\|y^{\prime}\right\|^{2}+\frac{p}{q} y^{2}(1)+\frac{r}{s} y^{2}(0) \leq & \frac{B}{q} y(1)+\frac{A}{s} y(0)-a \lambda\|y\|^{2}+|b| \int_{0}^{1}|y(t)|\left|y^{\prime}(t)\right| d t \\
& +\int_{0}^{1}|y(t)|\left|h\left(t, y(t), y^{\prime}(t)\right)\right| d t .
\end{aligned}
$$

Following the ideas of Theorem 2.1 we now obtain

$$
\begin{aligned}
\left\|y^{\prime}\right\|^{2}+\frac{p}{q} y^{2}(1)+\frac{r}{s} y^{2}(0) \leq & \frac{B}{q} y(1)+\frac{A}{s} y(0)-a \lambda\|y\|^{2}+\mid b\|\| y\|\| y^{\prime} \| \\
& +\frac{\varepsilon}{2}\|y\|^{2}+\frac{2 M^{2}}{\varepsilon}\left\{\|y\|^{2 \alpha}+\left\|y^{\prime}\right\|^{2 \beta}+1\right\} .
\end{aligned}
$$

Lemma 2.3(ii) and Lemma 2.4 imply

$$
\begin{aligned}
\left\|y^{\prime}\right\|^{2} & +\frac{p}{q} y^{2}(1)+\frac{r}{s} y^{2}(0) \\
\leq & -a \lambda\|y\|^{2}+\frac{B}{q} y(1)+\frac{A}{s} y(0)+|b|\left\|y^{\prime}\right\|\left\{\frac{2}{\pi}\left\|y^{\prime}\right\|+\frac{1}{2}[y(0)+y(1)]\right\} \\
& +\frac{\varepsilon}{2}\left\{\left(\frac{\sqrt{2}}{(\sqrt{2}-1)}\right) \frac{1}{\pi^{2}}\left\|y^{\prime}\right\|^{2}\left(\frac{2-\sqrt{2}}{2(\sqrt{2}-1)}\right)\left[y^{2}(0)+y^{2}(1)\right]\right\} \\
& +2 \frac{M^{2}}{\varepsilon}\left\{1+\left\|y^{\prime}\right\|^{2 \beta}+2^{2 \alpha}\left[\left(\frac{2}{\pi}\right)^{2 \alpha}\left\|y^{\prime}\right\|^{2 \alpha}+\left(\frac{1}{2}\right)^{2 \alpha}\left(|y(0)|^{2 \alpha}+|y(1)|^{2 \alpha}\right)\right]\right\} .
\end{aligned}
$$


That is

$$
\begin{aligned}
\left\|y^{\prime}\right\|^{2}+ & \frac{p}{q} y^{2}(1)+\frac{r}{s} y^{2}(0) \\
\leq & -a \lambda\|y\|^{2}+\frac{B}{q} y(1)+\frac{A}{s} y(0)+\frac{2}{\pi}|b|\left\|y^{\prime}\right\|^{2}+\frac{1}{2}|b|\left\{\frac{|y(0)|^{2}}{2}+\frac{\left\|y^{\prime}\right\|^{2}}{2}\right\} \\
& +\frac{1}{2}|b|\left\{\frac{|y(1)|^{2}}{2}+\frac{\left\|y^{\prime}\right\|^{2}}{2}\right\} \\
& +\frac{\varepsilon}{2}\left\{\left(\frac{\sqrt{2}}{\sqrt{2}-1}\right) \frac{1}{\pi^{2}}\left\|y^{\prime}\right\|^{2}+\frac{2-\sqrt{2}}{2(\sqrt{2}-1)}\left[y^{2}(0)+y^{2}(1)\right]\right\} \\
& +\frac{2 M^{2}}{\varepsilon}\left\{1+\left\|y^{\prime}\right\|^{2 \beta}+2^{2 \alpha}\left[\left(\frac{2}{\pi}\right)^{2 \alpha}\left\|y^{\prime}\right\|^{2 \alpha}+\left(\frac{1}{2}\right)^{2 \alpha}\left(|y(0)|^{2 \alpha}+|y(1)|^{2 \alpha}\right)\right]\right\} .
\end{aligned}
$$

Consequently

$$
\begin{aligned}
(1 & \left.-|b|\left(\frac{2}{\pi}+\frac{1}{2}\right)-\frac{\sqrt{2} \varepsilon}{2 \pi^{2}(\sqrt{2}-1)}\right)\left\|y^{\prime}\right\|^{2}+\left(\frac{p}{q}-\frac{|b|}{4}-\frac{(2-\sqrt{2}) \varepsilon}{4(\sqrt{2}-1)}\right) y^{2}(1) \\
& +\left(\frac{r}{s}-\frac{|b|}{4}-\frac{(2-\sqrt{2}) \varepsilon}{4(\sqrt{2}-1)}\right) y^{2}(0) \\
\leq & -a \lambda\|y\|^{2}+\frac{B}{q} y(1)+\frac{A}{s} y(0) \\
& +\frac{2 M^{2}}{\varepsilon}\left\{1+\left\|y^{\prime}\right\|^{2 \beta}+2^{2 \alpha}\left[\left(\frac{2}{\pi}\right)^{2 \alpha}\left\|y^{\prime}\right\|^{2 \alpha}+\left(\frac{1}{2}\right)^{2 \alpha}\left(|y(0)|^{2 \alpha}+|y(1)|^{2 \alpha}\right)\right]\right\} .
\end{aligned}
$$

Consider case $(*)$ with $a \geq 0$. We can delete the $a \lambda$ term from (2.11) and then fix $\varepsilon>0$ suitably small so that the terms in brackets on the left-hand side of (2.11) are positive. Note also if $L \geq 0,0 \leq k<2,0<h<1$ are given constants then there exists a constant $N>0$ such that $L x^{k} \leq h x^{2} / 2+N$ for all $x \geq 0$. This together with (2.11) implies there is a constant $C_{1}$ independent of $\lambda$ such that

$$
\begin{aligned}
\frac{1}{2}(1 & \left.-|b|\left(\frac{2}{\pi}+\frac{1}{2}\right)-\frac{\sqrt{2} \varepsilon}{4(\sqrt{2}-1)}\right)\left\|y^{\prime}\right\|^{2}+\frac{1}{2}\left(\frac{p}{q}-\frac{|b|}{4}-\frac{(2-\sqrt{2}) \varepsilon}{4(\sqrt{2}-1)}\right) y^{2}(1) \\
& +\frac{1}{2}\left(\frac{r}{s}-\frac{|b|}{4}-\frac{(2-\sqrt{2}) \varepsilon}{4(\sqrt{2}-1)}\right) y^{2}(0) \leq C_{1} .
\end{aligned}
$$

Consequently there exists a constant $M_{1}$ independent of $\lambda$ such that

$$
\left\|y^{\prime}\right\| \leq M_{1}, \quad|y(0)| \leq M_{1} \text { and }|y(1)| \leq M_{1} .
$$

On the other hand consider case $(* *)$ with $a<0$. Thus

$$
-a \lambda\|y\|^{2} \leq-a\|y\|^{2} \leq \frac{-a \sqrt{2}}{\pi^{2}(\sqrt{2}-1)}\left\|y^{\prime}\right\|^{2}-\frac{a(2-\sqrt{2})}{2(\sqrt{2}-1)}\left[y^{2}(0)+y^{2}(1)\right] .
$$


Substitute this into (2.11) to obtain

(2.13)

$$
\begin{aligned}
(1- & |b| \\
& \left.\left.+\left(\frac{2}{\pi}+\frac{1}{2}\right)+\frac{a}{\pi^{2}}\left(\frac{\sqrt{2}}{\sqrt{2}-1}\right)-\frac{\sqrt{2} \varepsilon}{2 \pi^{2}(\sqrt{2}-1)}\right) \| \frac{a(2-\sqrt{2})}{2(\sqrt{2}-1)}-\frac{(2-\sqrt{2}) \varepsilon}{4(\sqrt{2}-1)}\right) y^{\prime} \|^{2}(1) \\
& +\left(\frac{r}{s}-\frac{|b|}{4}+\frac{a(2-\sqrt{2})}{2(\sqrt{2}-1)}-\frac{(2-\sqrt{2}) \varepsilon}{4(\sqrt{2}-1)}\right) y^{2}(0) \\
\leq & \frac{B}{q} y(1)+\frac{A}{s} y(0) \\
& +\frac{2 M^{2}}{\varepsilon}\left\{1+\left\|y^{\prime}\right\|^{2 \beta}+2^{2 \alpha}\left[\left(\frac{2}{\pi}\right)^{2 \alpha}\left\|y^{\prime}\right\|^{2 \alpha}+\left(\frac{1}{2}\right)^{2 \alpha}\left(|y(0)|^{2 \alpha}+|y(1)|^{2 \alpha}\right]\right\} .\right.
\end{aligned}
$$

Fix $\varepsilon>0$ suitably small so that the terms in brackets on the left-hand side of (2.13) are positive and as above this leads to (2.12) for a certain constant $M_{1}$ independent of $\lambda$ in $(0,1)$.

From (2.12) we have that $|y(t)| \leq|y(0)|+\left|\int_{0}^{t} y^{\prime}(s) d s\right| \leq M_{1}+\left\|y^{\prime}\right\| \leq 2 M_{1} \equiv$ $M_{0}$ for all $t \in[0,1]$, which is the required a priori bound on $y(t)$. Also from (i) and (ii) we can determine constants $E$ and $F$ such that

$$
\left|f\left(t, y(t), y^{\prime}(t)\right)\right| \leq E\left|y^{\prime}(t)\right|^{2}+F .
$$

Consequently $\int_{0}^{1}\left|y^{\prime \prime}(t)\right| d t \leq E\left\|y^{\prime}\right\|^{2}+F \leq E M_{1}^{2}+F \equiv M_{2}$ and so

$$
\left|y^{\prime}(t)\right| \leq\left|y^{\prime}(0)\right|+\int_{0}^{1}\left|y^{\prime \prime}(t)\right| d t \leq \frac{|A|+r M_{1}}{s}+M_{2}
$$

for $t \in[0,1]$ and the result follows.

Remark. It is possible to extend all the existence results in this section to systems of second order boundary value problems.

\section{HIGHER ORDER EQUATIONS}

The ideas in $\S 2$ may be extended to higher order equations, in particular to third and fourth order boundary value problems. Since the technique is similar we will for the sake of brevity restrict our discussion to the fourth order problem:

$$
\left\{\begin{array}{l}
y^{\mathrm{iv}}=f\left(t, y, y^{\prime}, y^{\prime \prime}, y^{\prime \prime \prime}\right), \quad 0<t<1, \\
y(0)=A, y(1)=B, y^{\prime \prime}(0)=C, y^{\prime \prime}(1)=D,
\end{array}\right.
$$

with $f:[0,1] \times \mathbb{R}^{4} \rightarrow \mathbb{R}$ continuous. Our results improve upon those in $[9,14]$.

Making the change of variable $w(t)=y(t)-\eta(t)$, where $\eta(t)=\frac{1}{3}(D-C) t^{3}+$ $\frac{1}{2} C t^{2}+\left\{(B-A)-\frac{1}{6}(D+2 C)\right\} t+A$, we notice that if $y$ is a solution to (3.1) 
then $w(t)$ satisfies

$$
\left\{\begin{array}{l}
w^{\text {iv }}=f\left(t, w+\eta, w^{\prime}+\eta^{\prime}, w^{\prime \prime}+\eta^{\prime \prime}, w^{\prime \prime \prime}+\eta^{\prime \prime \prime}\right), \quad 0<t<1, \\
w(0)=w(1)=w^{\prime \prime}(0)=w^{\prime \prime}(1)=0 .
\end{array}\right.
$$

Theorem 3.1. Assume that $f$ has the decomposition $f(t, u, v, z, s)=$ $g(t, u, v, z, s)+h(t, u, v, z, s)$ such that

(i) $z g(t, u, v, z, s) \geq a|z|^{2}+b|z v|+c|z u|+d z s+f|z s|$ for $(t, u, v, z, s)$ $\in[0,1] \times \mathbb{R}^{4}$ and $|g(t, u, v, z, s)| \leq C(t, u, v, z)|s|^{2}+D(t, u, v, z)$ where $C$ and $D$ are bounded on bounded sets.

(ii) there exists constants $K \geq 0,0 \leq m, n, p, q<2$ such that $[(D-C) t+C] g(t, u, v, z, s) \leq K\left\{|u|^{m}+|v|^{n}+|z|^{p}+|s|^{q}+1\right\}$ for $(t, u, v, z, s) \in[0,1] \times \mathbb{R}^{4}$.

(iii) $|h(t, u, v, z, s)| \leq M\left\{|u|^{\alpha}+|v|^{\beta}+|z|^{\sigma}+|s|^{r}+1\right\}$ for constants $M, \alpha, \beta$, $\sigma$, and $r$ with $0 \leq \alpha, \beta, \sigma, r<1$.

Then (3.2) (and consequently (3.1)) has a $C^{4}[0,1]$ solution in each of the following cases:

(*) $a \geq 0, b \leq 0, c \leq 0, f \leq 0$, and $|c|+|f| \pi^{3}<\pi^{4}+b \pi$;

(**) $a \leq 0, b \leq 0, c \leq 0, f \leq 0$, and $|c|+|f| \pi^{3}<\pi^{4}+a \pi^{2}+b \pi$;

$(* * *) \quad a \leq 0, b \geq 0, c \leq 0, f \leq 0$, and $|c|+|f| \pi^{3}<\pi^{4}+a \pi^{2}$.

Remark. There are obvious results also for the other combinations of signs of $a, b, c$, and $f$.

Proof. Let $w(t)$ be a solution to

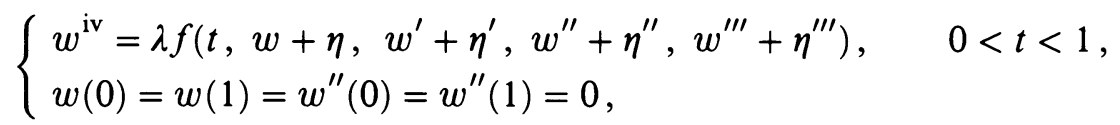

where $0<\lambda<1$. Now $\int_{0}^{1} w^{\prime \prime}(t) w^{i v}(t) d t=-\left\|w^{\prime \prime \prime}\right\|^{2}$ and so we have

$$
\begin{aligned}
\left\|w^{\prime \prime \prime}\right\| \leq & -\lambda \int_{0}^{1}\left(w^{\prime \prime}+\eta^{\prime \prime}\right) g\left(t, w+\eta, w^{\prime}+\eta^{\prime}, w^{\prime \prime}+\eta^{\prime \prime}, w^{\prime \prime \prime}+\eta^{\prime \prime \prime}\right) d t \\
& +\lambda \int_{0}^{1} \eta^{\prime \prime} g\left(t, w+\eta, w^{\prime}+\eta^{\prime}, w^{\prime \prime}+\eta^{\prime \prime}, w^{\prime \prime \prime}+\eta^{\prime \prime \prime}\right) d t \\
& +\int_{0}^{1}\left|w^{\prime \prime}\right|\left|h\left(t, w+\eta, w^{\prime}+\eta^{\prime}, w^{\prime \prime}+\eta^{\prime \prime}, w^{\prime \prime \prime}+\eta^{\prime \prime \prime}\right)\right| d t .
\end{aligned}
$$

Cauchy's inequality, condition (iii), and Hölder's inequality now implies (3.4)

$$
\begin{aligned}
& \int_{0}^{1}\left|w^{\prime \prime} \| h\left(t, w+\eta, w^{\prime}+\eta^{\prime}, w^{\prime \prime}+\eta^{\prime \prime}, w^{\prime \prime \prime}+\eta^{\prime \prime \prime}\right)\right| d t \\
& \quad \leq \frac{\varepsilon}{2}\left\|w^{\prime \prime}\right\|^{2}+\frac{8 M^{2}}{\varepsilon}\left\{2^{2 \alpha}\|w\|^{2 \alpha}+2^{2 \beta}\left\|w^{\prime}\right\|^{2 \beta}+2^{2 \sigma}\left\|w^{\prime \prime}\right\|^{2 \sigma}+2^{2 r}\left\|w^{\prime \prime \prime}\right\|^{2 r}+C_{1}\right\}
\end{aligned}
$$

for some constant $C_{1}$ independent of $\lambda$. 
On the other hand, conditions (i) and (ii) and Hölder's integral inequality yields

$$
\begin{aligned}
G(\lambda)= & -\lambda \int_{0}^{1}\left(w^{\prime \prime}+\eta^{\prime \prime}\right) g\left(t, w+\eta, w^{\prime}+\eta^{\prime}, w^{\prime \prime}+\eta^{\prime \prime}, w^{\prime \prime \prime}+\eta^{\prime \prime \prime}\right) d t \\
& +\lambda \int_{0}^{1} \eta^{\prime \prime} g\left(t, w+\eta, w^{\prime}+\eta^{\prime}, w^{\prime \prime}+\eta^{\prime \prime}, w^{\prime \prime \prime}+\eta^{\prime \prime \prime}\right) d t \\
\leq & -a \lambda\|w\|^{2}+2|a| K_{2}\left\|w^{\prime \prime}\right\|+|a| K_{2}-b \lambda \int_{0}^{1}\left|w^{\prime}(t) w^{\prime \prime}(t)\right| d t \\
& +|b| K_{1}\left\|w^{\prime \prime}\right\|+|b| K_{2}\left\|w^{\prime}\right\|+|b| K_{1} K_{2}+|c|\|w\|\left\|w^{\prime \prime}\right\|+|c| K_{2}\|w\| \\
& +|c| K_{0}\left\|w^{\prime \prime}\right\|+|c| K_{2} K_{0}+|d| K_{2}\left\|w^{\prime \prime \prime}\right\|+|d| K_{3}\left\|w^{\prime \prime}\right\|+|d| K_{2} K_{3} \\
& +|f|\left\|w^{\prime \prime}\right\|\left\|w^{\prime \prime \prime}\right\|+|f| K_{2}\left\|w^{\prime \prime \prime}\right\|+|f| K_{3}\left\|w^{\prime \prime}\right\|+|f| K_{2} K_{3} \\
& +K\left\{2^{m}\|w\|^{m}+2^{n}\left\|w^{\prime}\right\|^{n}+2^{p}\left\|w^{\prime \prime}\right\|^{p}+2^{q}\left\|w^{\prime \prime \prime}\right\|^{q}+C_{2}\right\}
\end{aligned}
$$

for some constant $C_{2}$; here $K_{j}=\max _{[0,1]}\left|\eta^{(j)}(t)\right|, j=0,1,2,3$. Let $E=$ $2|a| K_{2}+|b| K_{1}+|c| K_{0}+(|d|+|f|) K_{3}, F=|b| K_{2}, G=|c| K_{2}, H=(|d|+|f|) K_{2}$, $I=|a| K_{2}+|b| K_{1} K_{2}+|c| K_{2} K_{0}+(|d|+|f|) K_{2} K_{3}$ and consequently

$$
\begin{aligned}
G(\lambda) \leq & -a \lambda\|w\|^{2}-b \lambda \int_{0}^{1}\left|w^{\prime}(t) w^{\prime \prime}(t)\right| d t+|c|\|w\|\left\|w^{\prime \prime}\right\| \\
& +|f|\left\|w^{\prime \prime}\right\|\left\|w^{\prime \prime \prime}\right\|+E\left\|w^{\prime \prime}\right\|+F\left\|w^{\prime}\right\|+G\|w\|+H\left\|w^{\prime \prime \prime}\right\| \\
& +I+K\left\{2^{m}\|w\|^{m}+2^{n}\left\|w^{\prime}\right\|^{n}+2^{p}\left\|w^{\prime \prime}\right\|^{p}+2^{q}\left\|w^{\prime \prime \prime}\right\|^{q}+C_{2}\right\} .
\end{aligned}
$$

Substitute (3.4) and (3.5) in (3.3) to obtain

$$
\begin{aligned}
\left\|w^{\prime \prime \prime}\right\|^{2} \leq & -a \lambda\|w\|^{2}-b \lambda \int_{0}^{1}\left|w^{\prime}(t) w^{\prime \prime}(t) d t+\right| c\left|\|w\|\left\|w^{\prime \prime}\right\|+\right| f \mid\left\|w^{\prime \prime}\right\|\left\|w^{\prime \prime \prime}\right\| \\
& +\frac{\varepsilon}{2}\left\|w^{\prime \prime}\right\|^{2}+E\left\|w^{\prime \prime}\right\|+F\left\|w^{\prime}\right\|+G\|w\|+H\left\|w^{\prime \prime \prime}\right\|+I \\
& +K\left\{2^{m}\|w\|^{m}+2^{n}\left\|w^{\prime}\right\|^{n}+2^{p}\left\|w^{\prime \prime}\right\|^{p}+2^{q}\left\|w^{\prime \prime \prime}\right\|^{q}+C_{2}\right\} \\
& +\frac{8 M^{2}}{\varepsilon}\left\{2^{2 \alpha}\|w\|^{2 \alpha}+2^{2 \beta}\left\|w^{\prime}\right\|^{2 \beta}+2^{2 \sigma}\left\|w^{\prime \prime}\right\|^{2 \sigma}+2^{2 r}\left\|w^{\prime \prime \prime}\right\|^{2 r}+C_{1}\right\} .
\end{aligned}
$$

Theorem 1.2 now implies

$$
\begin{aligned}
(1- & \left.\frac{|c|}{\pi^{4}}-\frac{|f|}{\pi}-\frac{\varepsilon}{2 \pi^{2}}\right)\left\|w^{\prime \prime \prime}\right\|^{2} \\
\leq & -a \lambda\left\|w^{\prime \prime}\right\|^{2}-b \lambda \int_{0}^{1}\left|w^{\prime}(t) w^{\prime \prime}(t)\right| d t+\left[\frac{E}{\pi}+\frac{F}{\pi^{2}}+\frac{G}{\pi^{3}}\right]\left\|w^{\prime \prime \prime}\right\|+I \\
& +K\left\{\frac{2^{m}}{\pi^{3 m}}\left\|w^{\prime \prime \prime}\right\|^{m}+\frac{2^{n}}{\pi^{2 n}}\left\|w^{\prime \prime \prime}\right\|^{n}+\frac{2^{p}}{\pi^{p}}\left\|w^{\prime \prime \prime}\right\|^{p}+2^{q}\left\|w^{\prime \prime \prime}\right\|^{q}+C_{2}\right\} \\
& +\frac{8 M^{2}}{\varepsilon}\left\{\frac{2^{2 \alpha}}{\pi^{6 \alpha}}\left\|w^{\prime \prime \prime}\right\|^{2 \alpha}+\frac{2 \beta}{\pi^{4 \beta}}\left\|w^{\prime \prime \prime}\right\|^{2 \beta}+\frac{2^{2 \sigma}}{\pi^{2 \sigma}}\left\|w^{\prime \prime \prime}\right\|^{2 \sigma}+2^{2 r}\left\|w^{\prime \prime \prime}\right\|^{2 r}+C_{1}\right\} .
\end{aligned}
$$


Consider case $(*)$ with $a \geq 0, b \leq 0$. We can delete the $a \lambda$ term from (3.6) and because $b \leq 0$ we have

$$
-b \lambda \int_{0}^{1}\left|w^{\prime}(t) w^{\prime \prime}(t)\right| d t \leq-b\left\|w^{\prime}\right\|\left\|w^{\prime \prime}\right\| \leq-\frac{b}{\pi^{3}}\left\|w^{\prime \prime \prime}\right\|^{2} .
$$

Substitute this into (3.6) to obtain on the left-hand side

$$
\left(1-\frac{|c|}{\pi^{4}}-\frac{|f|}{\pi}+\frac{b}{\pi^{3}}-\frac{\varepsilon}{2 \pi^{2}}\right)\left\|w^{\prime \prime \prime}\right\|^{2} .
$$

Now fix $\varepsilon>0$ suitably small so that the term in brackets is positive and thus there exists a constant $M_{3}$ independent of $\lambda$ such that

$$
\left\|w^{\prime \prime \prime}\right\| \leq M_{3}
$$

for any solution $w$ to $\left(3.2_{\lambda}\right)$.

Now consider case $(* *)$ with $a \leq 0$ and $b \leq 0$. We have

$$
-a \lambda\left\|w^{\prime \prime}\right\|^{2} \leq-a\left\|w^{\prime \prime}\right\|^{2} \leq-\frac{a}{\pi^{2}}\left\|w^{\prime \prime \prime}\right\|^{2} .
$$

Substitute (3.7) and (3.9) into (3.6) to obtain on the left-hand side

$$
\left(1-\frac{|c|}{\pi^{4}}-\frac{|f|}{\pi}+\frac{b}{\pi^{3}}+\frac{a}{\pi^{2}}-\frac{\varepsilon}{2 \pi^{2}}\right)\left\|w^{\prime \prime \prime}\right\|^{2} .
$$

Fix $\varepsilon>0$ suitably small so that the term in brackets is positive and as above this leads to (3.8) for a certain constant $M_{3}$ independent of $\lambda$ in $(0,1)$.

Finally, consider case $(* * *)$ with $a \leq 0$ and $b \geq 0$. We can delete the $-b \lambda$ term from (3.6). Substitute (3.9) into (3.6) to obtain on the left-hand side

$$
\left(1-\frac{|c|}{\pi^{4}}-\frac{|f|}{\pi}+\frac{a}{\pi^{2}}-\frac{\varepsilon}{2 \pi^{2}}\right)\left\|w^{\prime \prime \prime}\right\|^{2} .
$$

Now fix $\varepsilon>0$ suitably small so that the term in brackets is positive and this leads to (3.8).

From (3.8) and Hölder's inequality we have $|w|_{2} \leq M_{3}$ for any solution $w$ to $\left(3.2_{\lambda}\right)$. Also there exists constants $R$ and $S$ such that

$$
\mid f\left(t, w+\eta, w^{\prime}+\eta^{\prime}, w^{\prime \prime}+\eta^{\prime \prime}, w^{\prime \prime \prime}+\left.\eta^{\prime \prime \prime}|\leq R| w^{\prime \prime \prime}\right|^{2}+S .\right.
$$

Consequently

$$
\left|w^{\prime \prime \prime}(t)\right| \leq \int_{0}^{1}\left|w^{\mathrm{iv}}(t)\right| d t \leq R\left\|w^{\prime \prime \prime}\right\|^{2}+S \leq R M_{3}^{2}+S \equiv M_{4}
$$

for $t \in[0,1]$. All the required a priori bounds are obtained and therefore a solution to (3.2) exists. 


\section{REFERENCES}

1. A. R. Aftabizadeh, C. P. Gupta, and J. M. Xu, Existence and uniqueness theorems for three-point boundary value problems, SIAM J. Math. Anal. 20 (1989), 716-726.

2. L. E. Bobisud and Y. S. Lee, Existence for a class of nonlinear singular boundary value problems, Applicable Anal. (to appear).

3. L. E. Bobisud, D. O'Regan, and W. D. Royalty, Singular boundary value problems, Applicable Anal. 23 (1986), 233-243.

4. J. Dugundji and A. Granas, Fixed point theory, Monograf. Math. PWN, Warsaw, 1982.

5. M. Frigon and D. O'Regan, On a generalization of a theorem of S. Bernstein, Ann. Polon. Math. 48 (1988), 297-306.

6. A. Granas, R. B. Guenther, and J. W. Lee, Nonlinear boundary value problems for ordinary differential equations, Dissertationes Math. 244 PWN, Warsaw, 1985.

7. __ Existence principles for classical and caratheodory solutions of nonlinear systems and applications, Proc. Internat. Conf. on Theory and Appl. of Differential Equations, Ohio Univ. Press, Athens, 1988, 353-364.

8. __ Some general existence principles in the Carathéodory theory of nonlinear differential equations J. Math. Pure Appl. (to appear).

9. C. P. Gupta, Existence and uniqueness theorems for the bending of an elastic beam equation, Applicable Anal. 26 (1988), 289-304.

10. G. H. Hardy, J. E. Littlewood, and G. Polya, Inequalities, Cambridge Univ. Press, London and New York, 1952.

11. D. D. Hai, Existence and uniqueness of solution for a nonlinear second order differential equation in Hilbert space, Proc. Edinburgh Math. Soc. (2) 33 (1990), 89-95.

12. J. W. Lee and D. O'Regan, Existence of solutions to some initial value, two point and multipoint boundary value problems with discontinuous nonlinearities, Applicable Anal. 33 (1989), 57-77.

13. D. O'Regan, Second and higher order systems of boundary value problems, J. Math. Anal. Appl. (to appear).

14. Y. Yang, Fourth order two point boundary value problems, Proc. Amer. Math. Soc. 104 (1988), 175-180.

\section{Department of Mathematics, Maynooth College, Co. Kildare, Ireland}

Current address: Department of Mathematics, University College Galway, Galway, Ireland 